\title{
LEUCOAGGLUTINATION IN VITRO
}

Deepak Nayak. M, AshaPatil, Rachana Dhakal

1. Assistant Professor, Department of Pathology, Melaka Manipal Medical College, Manipal, Manipal University.

2. Assistant Professor, Department of Medical Laboratory Technology, Manipal College of Allied Health Sciences, Manipal University, Manipal.

3. Junior Resident, Department of Pathology, Melaka Manipal Medical College, Manipal, Manipal University.

\section{CORRESPONDING AUTHOR}

Dr. Deepak Nayak M.

Department of Pathology,

Melaka Manipal Medical College, Manipal University, Manipal -576104. India.

E-mail: deepzienator@gmail.com

Ph: 0091-9901920537.

ABSTRACT: Phenomenon such as cold agglutination of red blood cells and platelets are well recognized and extensively studied. Nevertheless, aggregation of leukocytes in vitro is a rare phenomenon and may result in pseudoleukopenia. The exact mechanism for this phenomenon has not been elucidated. We report one such rare case where a male patient with HIV presented with leucopenia. The analysis of ethylene diamine tetraacetic acid (EDTA) anticoagulated blood was done on Coulter LH 750 hematology analyzer. The Volume Conductivity Scatter (VCS) parameters and histogram generated were inconclusive .The peripheral smear revealed small aggregates of leucocytes. The leucoaggregates were reversed on incubating the blood sample at 37 degree Celsius for 30 minutes. This suggests the role of a temperature dependent antibody causing clumping of leucocytes; possibly in conjunction with EDTA.

KEY WORDS: leucoaggregates, EDTA, antibody, leucocytes.

INTRODUCTION: The increasing application of automated analyzers for routine hematological procedures has several advantages. But a possibility of spurious results is never out of bounds. They may be due to the interaction of blood cells with serum antibodies reacting with drugs or additives in reagents. Among these, platelet aggregation, neutrophil-platelet clumping (satellitosis), and aggregation of nucleated red cells, resulting in pseudo thrombocytopenia or pseudoleukocytosis have been repeatedly described.1,2,3 Aggregation of neutrophils in peripheral blood smears, also referred to as leucoaggregation, neutrophil agglutination granulocyte aggregation, or leukocyte clumping, is a very rare, mostly self-limiting phenomenon in patients. It has been reported sporadically in the hematological literature.1,3,4,5

In the majority of cases, malignancies, infections, or hepatic disorders have been identified as the underlying condition. The exact mechanism for neutrophil aggregation in vitro has not been elucidated. Its relation to the use of ethylene diamine tetra acetic acid (EDTA) as an anticoagulant has been described in all reports. While the role of cold antibodies in erythrocyte clumping is a recognized event, aggregation of neutrophils in vitro, by the same yardstick is only speculated. In such cases, the machine values are not an accurate reflection of the patient's condition. Thus, recognizing this phenomenon on the peripheral smear and correlating with the clinical parameters are necessary.

We present a unique dilemma, where a temperature dependent antibody could have been implicated for leucoagglutination. 
CASE REPORT: A 50 year old male patient, admitted to the hospital following psychiatric problems since 10 months. He had a significant past history of retroviral illness, diagnosed 10 years ago. He was placed on HAART therapy for the same. He also had bilateral cervical lymphadenopathy which was firm and matted. Remaining lymph nodes were not enlarged. Other systems were within normal limits on examination. The chest radiograph demonstrated multiple small opacities, suggestive of miliary tuberculosis.

Laboratory investigations revealed an elevated erythrocyte sedimentation rate (ESR) of $110 \mathrm{~mm}$ (1 hour). The complete blood counts (CBC) done on Coulter LH 750 hematology analyzer (Beckman Coulter, Fullerton, CA) showed a total leucocyte count of $2.9 \times 10^{3} /$ cu.mm. (Fig. 1)

The RBC and WBC histograms revealed the following data (Fig.2)

The total WBC count of $2.9 \times 10^{3}$ / cu.mm with a differential count of neutrophils (55\%), lymphocytes (30\%) and monocytes (12\%). The platelet count was $125 \times 10^{3} / \mathrm{cu} . \mathrm{mm}$.

The microscopic examination of the peripheral smear showed a normal total WBC as opposed to the leucopenia flagged by the analyzer. The neutrophils were seen as small aggregates, some as large clumps at the edges and the tail-end of the smear (Fig.3). The lymphocytes and the few monocytes were arranged discretely, suggesting that this phenomenon of clumping was restricted to neutrophils alone.

A repeat smear was assessed from the same sample to rule out technical errors such as poor spreading of the blood film on the glass slide which could cause artefactual aggregates of leucocytes. The microscopic findings of the repeat smear however, were consistent with the earlier smear.

The next step was warming the sample to 37 degree Celsius for $30 \mathrm{~min}$ and then repeating the smear. The smear revealed reversal of the clumping of the neutrophils, now seen as singly arranged cells. (Fig.3)

The clinician was requested to provide additional details so that we could explain the laboratory and smear findings. The serology tests (ELISA) revealed that the patient was seropositive for HIV. The $\mathrm{C}$ reactive protein (CRP) was elevated $(62 \mathrm{mg} / \mathrm{L})$. The liver function tests and renal function tests were normal. This data along with the smear findings prompted us to consider the role of autoantibodies.

We requested a serological study of antibodies, which showed an increase in IgM levels. After a review of the available literature, we suggested a possibility of an antibody-mediated leucocyte clumping and the subsequent pseudoleukopenia

DISCUSSION: The aggregation of leukocytes in vitro is a rare hematological phenomenon, and most reports in literature are based on single cases studies. This phenomenon may relate to malignancies, infections, hepatic disorders or autoimmune diseases. ${ }^{7}$ This has also been noted in apparently healthy subjects as well. ${ }^{8}$ In the vast majority of reported cases of neutrophil aggregation, agglutination was confined to mature neutrophils, but rosetting of mature forms around immature neutrophils has been described as well as aggregation of neutrophils with platelets. ${ }^{6}$ The pattern of neutrophil agglutination may show considerable variation with small aggregates consisting of less than 10 cells as well as leukocyte clumps of more than 100 cells.

It is believed that the mechanism of leukocyte aggregation is mainly related to the use of K2 EDTA anticoagulant or is temperature-mediated.1,9,10,11 In our case, the EDTA was the anticoagulant used. This hints at a possibility of a form of K2EDTA-related, cold antibody of IgM type in the patient's serum. The serum IgM antibody titre was elevated in our case. The reversal 
of clumping of the neutrophils after warming the sample also underlines the temperature dependent nature of this reversible phenomenon.

It is also postulated that the formation of leucocyte aggregates is a time-dependent phenomenon. 812 Thus, the number and size of the aggregates of a particular patient are dependent on the elapsed time interval between drawing blood into EDTA and preparation of the blood smear and/or the analysis in the Coulter. ${ }^{8}$ The analysis of the sample in our case was conducted on in Coulter LH 750 hematology analyzer ( Beckman Coulter, Fullerton, CA) within 20 minutes of phlebotomy. The ensuing smear was prepared and reviewed within the next 30 minutes; thus showing a mixture of both small and large clumps of neutrophils. The repeat smear from the sample however showed a marginal increase in the size of the neutrophil aggregates; possibly testifying to the time dependent nature of the event.

In addition, the VCS parameters are expected to change when leukocyte aggregation occurs. The Coulter VCS technology establishes the WBC differential using 3 measurements: individual cell volume, high-frequency conductivity, and laser-light scatter. The WBC volume is measured using impedance; the cells are in their near-native state, and this gives a good indication of the cell volume as it circulates in the blood. ${ }^{13}$ When several leukocytes aggregate together, the volume of WBC increases and would not be counted or would only be counted as one. This is because the volume exceeded the range of WBCs and the measurement therefore could result in pseudoleukopenia. This principle also explains the spurious decrease in the total leucocyte count in our case.

One of the VCS parameters, Mean Neutrophil Volume (MNV) is also expected to increase with the increase in the extent of leukocyte aggregation. ${ }^{14}$ The maximum MNV in our case was 155 (normal range of MNV is 138.2 to 147.8) The Neutrophil Distribution Width (NDW) is a parameter that reflects the difference in size of neutrophils (normal range of NDW is $19.0 \pm 1.5$ ) and it increases in cases of acute infection, inflammation, leukemia, or malaria. ${ }^{15}$ When the leukocytes aggregated, the WBCs become variable in volume and NDW increased markedly as a result. The maximum in our case was 29.6. This differs greatly from the reported level of NDW in acute infection $(24.4 \pm 4.5)$ and reported level in malaria $(22.5 \pm 3.5)$. In our cases, the disproportionate increase of NDW suggests that it could be used as an adjunct indicator of leukocyte aggregation along with the unexplained low WBC counts.

It is also an established knowledge that HIV infection can induce both warm and cold autoantibodies; thus contributing to cytopaenias. ${ }^{16}$ Our case was tested positive for HIV; which could possibly account for the source of the IgM antibodies. So far, there is only one case report in which a Mycoplasma infection has been found concomitantly with granulocyte agglutination. ${ }^{17}$ Although there is consensus on a causative role of EDTA in the formation of leukocyte aggregates, the role of anti-IgM antibodies has been gradually gaining acceptance. ${ }^{17}$

CONCLUSION: Leucoagglutination is a rare phenomenon. But when it is noticed in routine medical practice, it can be a vital clue to the underlying disease. Thus it is incumbent on the hematopathologist to be aware of this event and identify the consequences and aetiology of this rare entity.

ABBREVIATIONS:

VCS- Volume Conductivity Scatter

K2-EDTA -Dipotassium ethylene diamine tetraacetic acid

HAART- Highly Active Anti-Retroviral Therapy 
CRP- $C$ reactive protein

MNV-Mean Neutrophil Volume

NDW-Neutrophil Distribution Width

\section{REFERENCES:}

1. Hillyer CD, Knopf AN, Berkman EM. EDTA-dependent leucoagglutination. Am J Clin Pathol 1990; 94:458-461.

2. Lesesve JF, Haristoy X, Thouvenin M, Latger-Cannard V, Buisine J, Lecompte T. Pseudoleukopenia due to in vitro leukocyte agglutination polynuclear neutrophils: experience of a laboratory, review of the literature and future management. Ann Biol Clin (Paris)2000; 58:417-424.

3. Savage RA. Analytic inaccuracy resulting from hematology specimen characteristics. Three cases of clinically misleading artifacts affecting white blood cell and platelet counts. Am J Clin Pathol 1989;92:295-299.

4. Epstein HD, Kruskall MS. Spurious leukopenia due to in vitro granulocyte aggregation. Am J Clin Pathol 1988;89:652-655.

5. Guibaud S, Plumet-Leger A, Frobert Y. Transient neutrophil aggregation in a patient with infectious mononucleosis. Am J Clin Pathol 1983; 80:883-884.

6. Claviez A, Horst HA, Santer R, Suttorp M. Neutrophil aggregates in a 13-year-old girl: a rare hematological phenomenon.Ann Hematol 2003; 82:251-253.

7. Moraglio D, Banfi G, Arnelli A. Association of pseudothrombocytopenia and pseudoleukopenia: Evidence for different pathogenic mechanisms. Scand J Clin Lab Invest 1994;54:257-265.

8. Lombarts, A. J. P. F., de Kieviet W. Recognition and prevention of pseudothrombocytopenia and concomitant pseudoleukocytosis. Am J Clin Pathol 1988;89:634-639.

9. Glasser L. Pseudo-neutropenia secondary to leukoagglutination. Am J Hematol 2005;80:147.

10. Carr ME, Whitehead J, Carlson P, et al. Case report: Immunoglobulin Mmediated, temperature-dependent neutrophil agglutination as a cause ofpseudoneutropenia. Am J Med Sci. 1996;311:92-95.

11. Lesesve JF, Haristoy X, Lecompte T. EDTA-dependent leucoagglutination. Clin Lab Hematol 2002;24:67-69.

12. Savage, R. A. (1984) Pseudoleukocytosis due to EDTA induced platelet clumping. Am J Clin Pathol 1984; 81:317-322.

13. Beckman Coulter. Coulter LH750 system: Operation principles. Operator's Guide. 2004:9.

14. Chaves F, Tierno B, Xu D. Quantitative determination of neutrophil VCS parameters by the Coulter automated hematology analyzer: New and reliable indicators for acute bacterial infection. Am J Clin Pathol 2005;124:440-444.

15. Bagdasaryan R, Zhou Z, Tierno B, et al. Neutrophil VCS parameters are superior indicators for acute infection. Lab Hematol 2007;13:12-16.

16. Deol I, Hernandez AM, Pierre RV.Ethylenediaminetetraacetic acid-associated leucoagglutination. Am J Clin Pathol 1995; 103:338-340. 
17. Robbins SH, Conly MA, Oettinger J. Cold-induced granulocyte agglutination. A cause of pseudoleukopenia. Arch Pathol Lab Med 1991; 115:155-157.

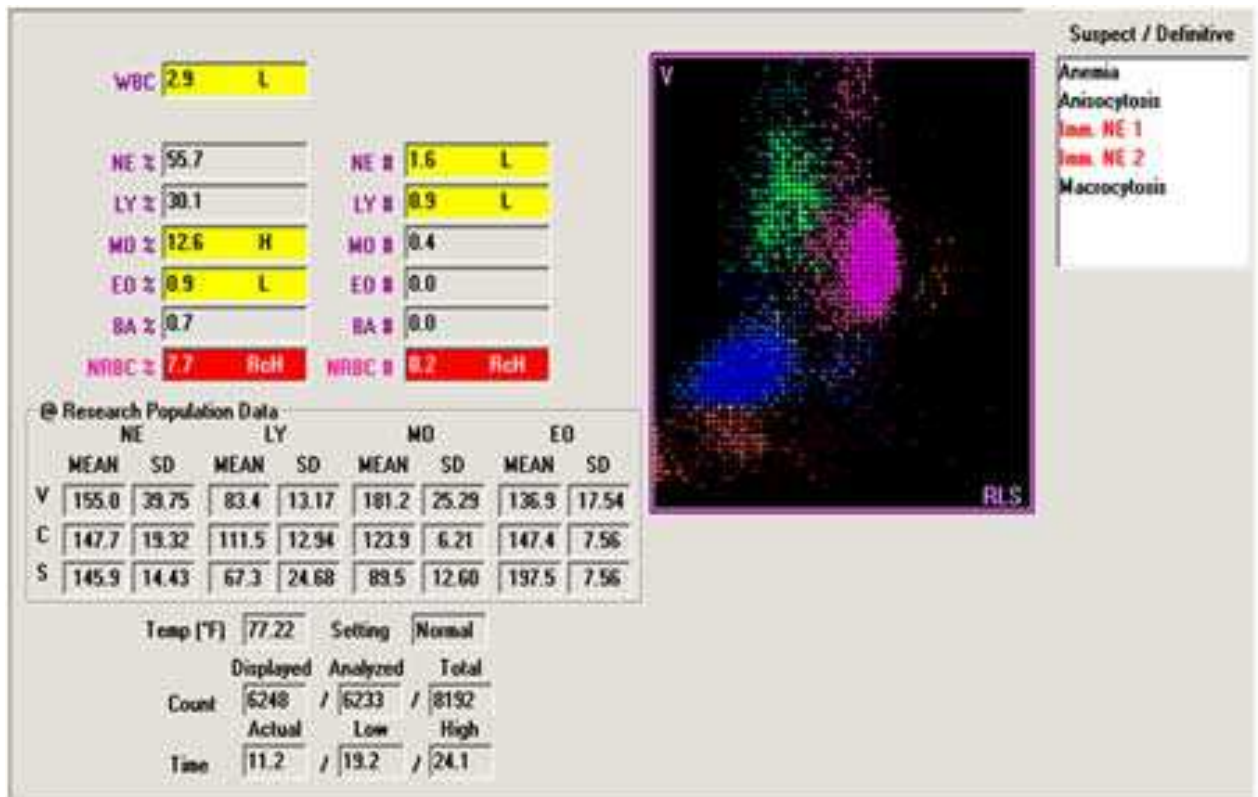

Fig.1: The VCS parameters in Coulter LH 750 hematology analyzer (Beckman Coulter, Fullerton, CA)

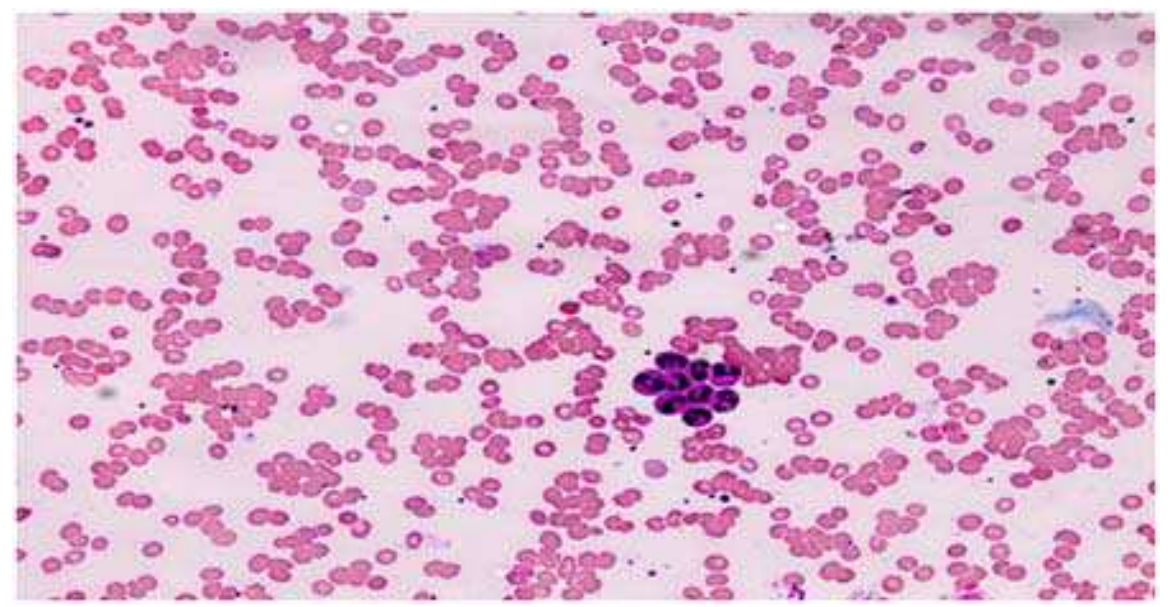

Fig.2: Peripheral smear showing small aggregates of leucocytes (Leishman; $x$ 100) 


\section{CASE REPORT}

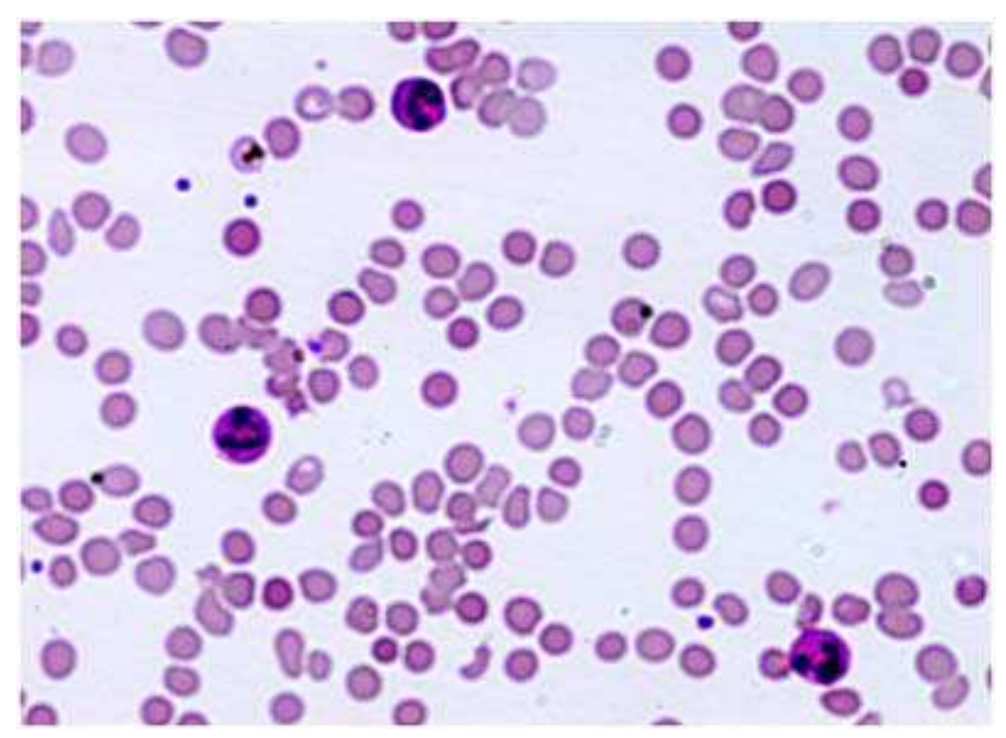

Fig.3: Peripheral smear showing discretely spread out leucocytes after warming the sample for $37^{\circ} \mathrm{C}$ for 30 minutes (Leishman; $\mathrm{x} 100$ ) 